(c)

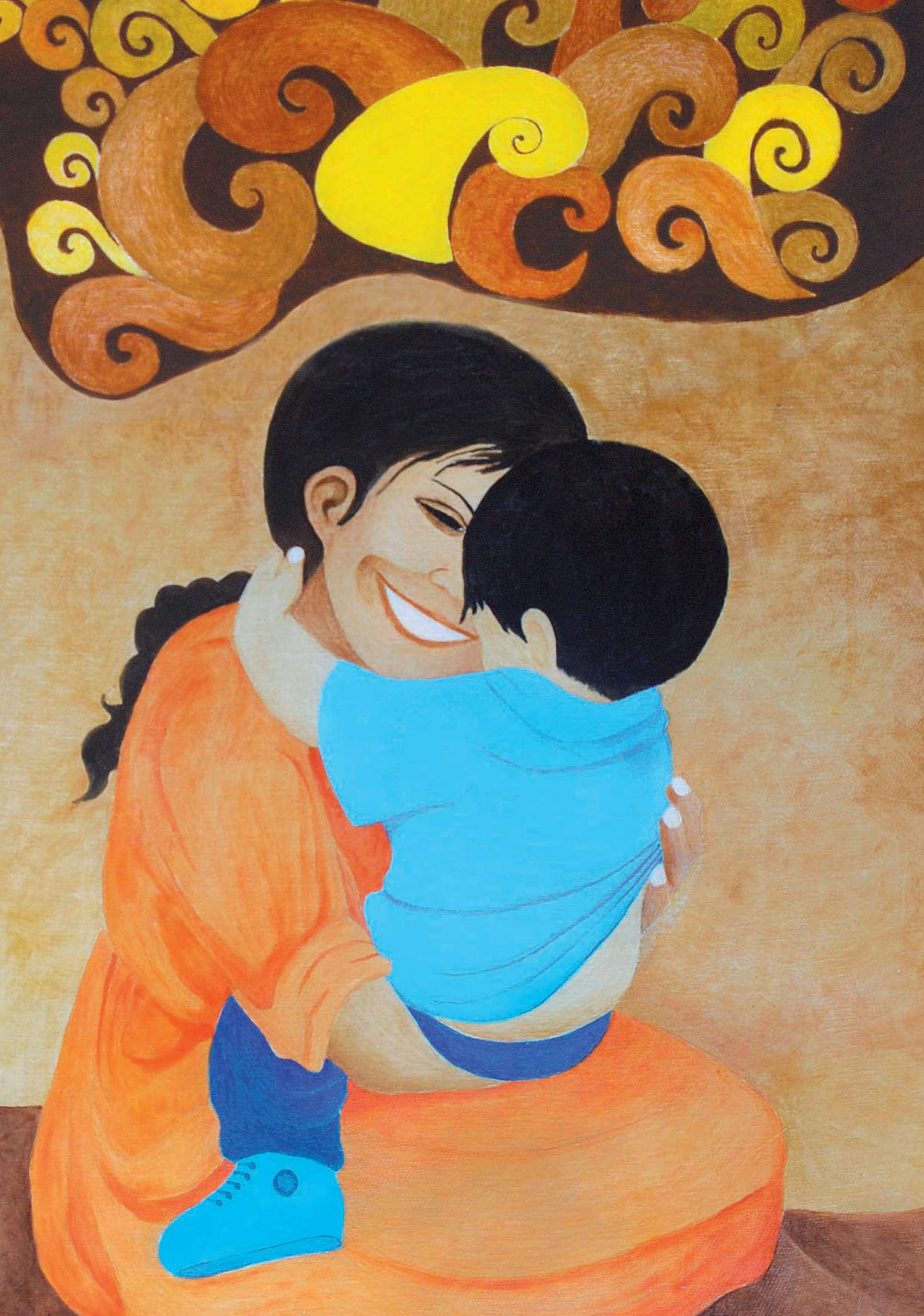




\section{Una demografía sentida: una nueva mirada en el sentir de los pueblos}

Alejandro Melgar Quiñonez*

RESUMEN. Los lugares poblados se fundan y se mueven con la lógica de la sobrevivencia, en ellos se forman las primarias estructuras sociales que adicionadas de sentires y experiencias, doblan páginas de historia y adquieren identidad y una forma propia de ver el mundo y su entorno natural. Estas poblaciones cargadas de valores se resisten ante contextos de pobreza y desigualdad social y, hoy se ven sumergidos en una nueva vorágine que los mueve hacia latitudes más hostiles e indiferentes. Una nueva mirada que trasciende en la demografía social y que está configurando un nuevo rostro humano. Quizás el dilema fundamental del mundo actual, sea la indiferencia que está pesando sobre el ser humano y su angustia, ante la pérdida de valores y sentimientos de pertenencia cultural; frente a un mercado que impone lógicas laborales y de consumo que están moviendo poblaciones por rutas inciertas, en la búsqueda de mejores condiciones de vida. Por ello la demografía no puede limitarse únicamente a contar eventos o poblaciones sino también a dar una interpretación de las dinámicas poblacionales que tienen su propia naturaleza y desarrollo en este devenir histórico, donde el sentir de los pueblos esta capturado por una globalización deshumanizada.

Palabras claves: población, tejido social, movilidad humana, dinamica demográfica.

ABSTRAC. Overview populated places merge and move with the logic of survival, they are formed primary social structures that add-on of feelings and experiences, folded pages of history and acquire identity and a way of seeing the world and its natural environment. These values-laden populations resist in contexts of poverty and social inequality, and today are immersed in a new vortex that moves them towards latitudes more hostile and indifferent. A new look that transcends the social demographics and that is setting a new human face.

Perhaps the fundamental dilemma of today's world, the indifference that is weighing on the human being and his anguish, against the loss of values and feelings of cultural belonging; facing a market that imposes labor logics and consumption that are moving populations by routes uncertain, in search of better living conditions. For this reason the demographics cannot be limited only to have events or populations but also to give an interpretation of population dynamics that have their own nature and development in this historic evolution, where people feel this captured a dehumanized globalization.

Keywords: population, social fabric, human mobility, demographic dynamics.

\section{Pioneros o iniciadores}

Los lugares poblados surgieron con la certeza de quienes fundaron en ellos la esperanza de tener un espacio posible. Habitantes que sembraron en esas geografías las posibilidades de reproducir la vida y darle sustento y razón a sus aspiraciones. En ellos se fue consolidando un sentir que determino formas de vida y significados que surgieron como producto de una realidad inicial que fue la suma y la satisfacción de muchas necesidades: una vivienda, un solar y una ubicación con nombre propio.

La mensura del área delimitada y constituida por su propio entorno natural, le otorgó un sentido propio a cada lugar. Esto dio origen a una gama de dominios o divisiones políticas administrativas que se iniciaron como caseríos, aldeas, cabeceras municipales y departamentales hasta llegar a conformar las grandes ciudades. Los primeros pobladores y futuros ciudadanos ${ }^{1}$, se fueron aglutinando de forma dispersa; pero en el afán de superar sus condiciones existenciales, comenzaron a reducir las distancias humanas, a tal grado de lograr una cohesión social que tuvo como marco integrador, el apoyo mutuo y la solidaridad.

1. Ser ciudadano no solo es reconocer los derechos por los aparatos del Estado a quienes nacieron en un territorio, sino también a las prácticas sociales y culturales que dan sentido de pertenencia y hace sentir diferentes a quienes poseen una misma lengua, semejantes formas de organizarse y satisfacer sus necesidades. (Canclini, Néstor; Consumidores y ciudadanos, 2009, pag 35) 
Este proceso dio origen a nuevas relaciones sociales que determinaron el sentido de pertenencia y arraigo hacia los bienes espirituales y materiales; lugares, hechos y situaciones tangibles e intangibles que constituyen el patrimonio vivo que les ha dado identidad cultural; una visión o bien una forma de ver el entorno humano y social. En aquellos remotos lugares, el tiempo paso y en la latitud de la memoria prevalecen aun los recuerdos de la tierra, los paisajes y las personas que colmaron de sujetos ancestrales el imaginario local.

El tejido social se fue haciendo cada vez más fuerte y complejo, la división social del trabajo fue inevitable y necesaria para que las aspiraciones y el devenir marcaran el ritmo y la dinámica de una cotidianidad que lograba cada día superar la sobrevivencia. La ruralidad marcaba el paso de una vida social que lejos de toda lógica natural, generaba sustento y sosiego a sus habitantes. La maquinaria del mercado apenas se asomaba para tender sus redes de perversidad y agotar las posibilidades de aquellas comunidades que producían un modo de vida basado en la agricultura y la artesanía y otras actividades primarias.

Hoy, en la distancia, los pueblos se ven en la vorágine de su historia ante las nuevas correlaciones económicas y sociales que están poniendo en riesgo la propia cultura. El impacto del orden mundial establecido está marcando nuevas líneas y nuevos propósitos. Las comunidades sometidas como objetos y victimas del consumo han tenido que migrar o sobrevivir ante las circunstancias impuestas por las desigualdades tanto sociales como territoriales, es decir, las que vienen con el tránsito de la pobreza y el subdesarrollo y que tuvieron origen en las relaciones sociales de producción impuestas durante la economía colonial y los nuevos desplazamientos espaciales como producto de los grandes emprendimientos económicos. Otro problema es la violencia organizada o la conflictividad impuesta que incide en una transición demográfica forzada que da la pauta a una nueva dinámica demográfica, ajena al crecimiento, en el ciclo natural de las poblaciones.

\section{El ensanchamiento de las fronteras}

Las crisis políticas y económicas, libradas en la región centroamericana como producto de los ajustes en los modelos de desarrollo y el expansionismo geopolítico de las transnacionales y del capital financiero a través de la globalización ${ }^{2}$ provocaron grandes cambios acompañados de crisis y desprendimientos poblacionales que configuraron y transformaron las sociedades. El primer desarraigo se dio en los hogares que tomaron distancia, rompiendo el tejido social familiar y comunitario

Estas transformaciones, se pueden establecer a través de tres ejes temáticos críticos que están delimitando los cambios demográficos. Estos son resultantes de una composición demográfica que se expande o que se mueve a razón de una sociedad que se desarrolla en la opulencia por un lado y no retorna a la naturaleza ni le devuelve el privilegio de la vida y por el otro, una sociedad que vive soterrada en sus propias necesidades y que se mueve en busca de mejorar sus condiciones de vida.

El primer eje; corresponde al crecimiento poblacional que ha diversificado la demanda y la oferta, situación que ha sido captada y concentrada por los intereses del mercado, cuyas directrices han provocado un consumo ${ }^{3}$ desmedido; sin sentido, ni contenido y que impone un ritmo de vida que no satisface necesidades sentidas sino creadas.

El segundo eje: tiene relación con el calentamiento global que año con año se intensifica en mayor grado, provocando el agotamiento a la naturaleza y el incremento de los desastres llamados naturales que se manifiestan a través de largos periodos de lluvias o por extensas sequias que ponen en riesgo la agricultura de subsistencia, incrementan el área del corredor seco y el incremento de la hambruna.

El tercer eje: corresponde a los grandes emprendi-

2. La globalización es una estrategia aplicada a los viejos problemas del capitalismo, nos referimos a sus perennes preocupaciones por crear nuevos mercados y consumidores (Quezada, Rodrigo; Globalización y deshumanización, 2006, pag 39)

3. El famoso informe sobre los límites del crecimiento, emitido por el club de Roma en 1972 advertía sobre aquellos peligros y en particular en un marco ideológico neomalthusiano sobre el agotamiento de los recursos naturales frente al crecimiento poblacional. (Mari, Eduardo; El ciclo de la tierra, 2000, pag 39) 
mientos económicos impulsados a través de la agricultura que utiliza grandes extensiones de tierra para la producción de biocombustibles, poniendo en riesgo la soberanía y la seguridad alimentaria. Por otro lado la industria extractiva de la minería en cualquiera de sus formas que no solo está consumiendo el entorno natural como el agua, sino además está dejando a muchas comunidades sin tierra.

En toda esta disparidad de situaciones y condiciones, existe un fuerte impacto sobre las culturas; formas de vida que se diseminan por los procesos de asimilación y comienzan a ser agredidas por la intolerancia de las fronteras. La migración en cadena toma auge y se levanta como una alternativa ante las necesidades materiales de una realidad que se debate entre el ser o tener. Estas nuevas formas de relaciones sociales de subsistencia están proponiendo también formas distintas de organización y sobrevivencia que están cambiando el rostro humano y social, extendiendo la geografía humana hacia nuevas fronteras culturales.

Con el ensanchamiento de los modelos de desarrollo las poblaciones se vieron en la necesidad de trasladarse en busca de atributos y oportunidades que les abrieran el camino hacia otras alternativas: mejoras salariales y un mínimo de estabilidad. El binomio de la necesidad y la satisfacción, generaron una movilidad humana y poblacional fundada en el ascenso y en el deseo de superar sus condiciones de vida.

En esa transición se dieron una serie de cambios que alteraron también el tejido humano y social. Poblaciones cargadas de experiencias, valores y símbolos que trascendían en la cotidianidad y en la visión de la vida, se vieron en la necesidad de renunciar a sus lugares de origen y fundar sociedades con una composición demográfica diferenciada por idiomas y dialectos que interactuaron y le dieron a estos espacios, un sentido y un carácter multicultural que creo nuevos escenarios de interculturalidad. ${ }^{4}$

4. La Interculturalidad se refiere al escenario de convivencia o de interrelación, de diversas culturas; sentires, saberes y formas de ver el mundo. comparten una misma latitud y un momento histórico (Diccionario de sociología, Pratt, Henry, 2010, pag 158)
Hoy el sur sigue caminando por las viejas rutas, con rumbo hacia el hemisferio norte; la pobreza y el hambre buscan atravesar las fronteras e insertarse en las grandes ciudades colmadas de indiferencia. Una remesa llega hasta el auxilio de una familia, mientras el valor agregado en las cifras del capital financiero sigue en ascenso, gracias a los ajustes estructurales, los tratados de libre comercio y a las concesiones a las transnacionales.

\section{Una nueva mirada, una nueva angustia}

El desplazamiento transfronterizo tiene un efecto psicosocial en el espacio interpersonal de las familias y comunidades. Nuevos patrones de consumo y de concebir la vida, están cambiando la naturaleza humana y social. El fenómeno de la transculturización está lacerando de forma perversa a los pueblos y su cultura e imponiendo una nueva visión que por una parte agrede los valores y las normas de estos y por otra trascienden con los nuevos patrones culturales que la globalización ha impuesto como estándares de vida y consumo.

A qué obedece todo este trasiego de situaciones; será que la cosmovisión o las formas de ver la vida no tienen suficiente sustento y fortaleza para elevar y mantener la autonomía y la existencialidad y, así mismo estar a la altura de una época de cambios o bien, de un cambio de época. $\mathrm{O}$ será que la matriz del modelo de desarrollo neoliberal sigue socavando las viejas formas de pensamiento social, pero que en el fondo, prevalece la vieja tesis de la acumulación a largo plazo que se inició con la revolución industrial y que hoy se pone en boga en los imponentes corredores del mercado que se mueve por la línea de los grandes emprendimientos económicos y sus proyectos extractivos.

Quizás aún no se tenga la certeza de una respuesta que divise en pretérito una salida, viendo las complejidades que conlleva esta situación, pero el germen de la contradicción establece una dialéctica que pone de manifiesto la existencia de una demografía cultural, diferenciada por desigualdades, que abren una nueva brecha en el horizonte de la angustia y el desasosiego de los migrantes. Porque, atravesar lugares, llegar a puntos 
muertos y esconderse tras el cerrojo de una frontera no ha sido fácil.

En Guatemala durante el conflicto armado interno muchas comunidades se movilizaron en busca de salvar la vida, años después retornaron pero ya no fueron los mismos, sus sueños y esperanzas se diluyeron en el silencio de una noche larga de fronteras con muros altos, cercos con alambres de púas y guardias con perros al asecho. Y bajo esa larga noche también perdieron la comunicación; la forma de sentir, hablar y de ser con el otro y la naturaleza.

En Honduras se habla y se proponen megaproyectos, como las ciudades modelos, donde la modernidad tomara auge y se vestirá de gala con la nueva generación tecnológica, caracterizada por ser de punta o de vanguardia y que tiene como fin generar mayores y mejores satisfactores; según rezan los instrumentos mediáticos que alzan la voz por los grandes avances de la era digital, el silicón, la fibra óptica y los circuitos integrados.

Sin embargo no hablan de las poblaciones o de las características que estas deben tener, para llenar los espacios habitacionales de estas ciudades que se pregonan como alternativas urbanas para una vida mejor. Por el contrario, se sienten los efectos de las comunidades locales que están siendo desplazadas de su territorialidad, física y cultural; sin respetar su patrimonio y su sentido de pertenencia

Donde quedara entonces, el dominio o el lugar poblado de estas comunidades que hoy tienen una geografía y una razón para seguir existiendo. Con un patrimonio universal que lo conforman: idiomas, dialectos, normas, valores y significados manifiestos de una visión del mundo que se fundó en la cotidianidad y en los saberes ancestrales que entre otros se resisten a desaparecer de esta variedad humana que se confronta y, en el dilema de tener, tiene la tendencia a homogenizarse y negar su proceso socio histórico.

\section{El sentir de los pueblos una lección por aprender}

Las poblaciones crecen por su ciclo natural o bien por las dinámicas migratorias que las evocan. Las poblaciones y sus culturas están cambiando, nada permanece estable, nuevos escenarios económicos y sociales están provocando nuevas necesidades y la estrategia es permanecer o resistir en el sistema, pero el dilema es como controlar el crecimiento poblacional natural y el movimiento de población. Puede que la lectura de este fenómeno trascienda más allá de cualquier política de estado o de un modelo simplista de desarrollo. La verdad de los números es más que alarmante ${ }^{5}$ conlleva a una reflexión sobre el contexto y el entorno donde están moviéndose e identificándose estas poblaciones.

Cada ser que se traslada de una latitud a otra, lleva consigo un libro de saberes y experiencias que cada comunidad va construyendo y grabando a lo largo de su devenir histórico. Sin embargo a pesar de toda la resistencia cultural, cada año existen menos dialectos e idiomas y esto constituye una pérdida en el acervo cultural universal, situación que está dejando como resultante un sentir de gramáticas congeladas y significados perdidos, víctimas de la indiferencia y la ignorancia.

Pero el problema no termina ahí, la interacción con otros idiomas y culturas dominantes, está provocando el cierre en los ciclos de las identidades, es decir existen procesos de asimilación que están dejando en la rezaga a la cultura de cada pueblo. El dilema se cierne cuando los pueblos dejan de hablar su idioma porque con ello, se pierde un rastro de vida, conocimientos y saberes que alimentan la diversidad humana y social y que hoy está en peligro de extinción.

En las comunidades indígenas del pueblo maya, hay jóvenes que reniegan de su cultura. Por ejemplo: prefieren aprender el idioma español antes que su idioma materno y, van a la escuela con el propósito leer y escribir, como herramientas suficientes para priorizar en el aprendizaje y práctica de otro idioma; el inglés. El problema es que estas nuevas generaciones están renunciando a su cultura; su base económica se está transformando y han aprendido que seguir sembrando la tierra es seguir viviendo en el

5. Los cálculos de los expertos aseguran que para el año 2020 la población mundial será de siete mil seiscientos millones de personas, un dato que debe ser tomado en cuenta por los diseñadores de políticas, ante este crecimiento poblacional (La Agenda Inconclusa, Andersen y Lorch, 2002,pag 57) 
ciclo de la pobreza; entonces sitúan sus sueños y expectativas al otro lado de la frontera norte.

La dinámica demográfica sigue siendo un termómetro del movimiento de las poblaciones y sus activos. Estas poblaciones están conformando una nueva composición demográfica cultural que está provocando cambios y diferencias en la demografía y en el espíritu de los pueblos que trascienden o desaparecen por el miedo, el hambre o la transculturización ${ }^{6}$ que implica la adopción de otros valores y significados.

El dilema que se perfila en el devenir lo constituye no solo la desigualdad manifiesta en la esperanza como indicador de vida, sino también, el bajo índice de bienestar que debería de incluir además, el derecho de ser, el derecho de soñar y el derecho a la alegría entre otros, para que no crezca el desencanto y se rompa el hilo de la memoria con la tierra y las comunidades que los vieron nacer y que las formas de ver y sentir la vida, sigan en la ruta de ser un patrimonio de la cultura universal, una diversidad humana y cultural manifiesta en cada expresión.

\section{A manera de conclusión}

- Nuevas correlaciones de fuerzas, variables y contextos se mueven en rededor de las comunidades; mientras la tecnología y el conocimiento crecen de forma exponencial, la humanidad apenas va superando su evolución social de forma lineal y quizás ahora, queda como tarea hacer una sistematización de los procesos sociohistórico que dieron origen a ese registro humano que hoy es conocimiento acumulado y que identifica y diferencia a una cultura.

6. Transculturización o transculturación; que significa el paso de elementos de una cultura a la otra o bien la interpenetración de una cultura a otra y en procesos muchas veces forzados o violentos (Lugan, Jean, Elementos para el análisis de los sistemas sociales, 1995, pag 225-226)

- Un teorema elemental por demostrar, se establece como síntesis o resumen en esta configuración demográfica y cultural; la dinámica sociodemográfica tarde o temprano produce cambios en la base poblacional y provoca impactos en la conciencia y en la forma de ver la vida. Cambios que por su naturaleza y complejidad humana y social podrían modificar para siempre la demografía socio cultural.

- En el nuevo escenario mundial, la demografía se está convirtiendo en el asidero de mayor importancia intelectual que está poniendo de manifiesto diversas situaciones humanas, socioeconómicas y cultuales; que requieren de nuevos paradigmas, ante una matemática de la incertidumbre que devela, que las poblaciones crecen, directamente proporcional a sus necesidades materiales e inversamente proporcional a sus posibilidades de desarrollo.

- Se puede decir que la movilidad natural o forzada que se da a través de la migración, no solo mueve seres humanos necesitados de mejores condiciones de vida, sino también con ellos se mueven formas de pensar y de ver el mundo; patrimonios de vida que trascienden en la experiencia misma por superar la realidad y ser un tránsito hacia el desarrollo en un mundo más humano y fraternal, en donde redunden el derecho a la alegría, el derecho de ser y sobre todo el derecho y privilegio de vivir. 